University of Nebraska - Lincoln

DigitalCommons@University of Nebraska - Lincoln

Faculty Publications: Materials Research

Science and Engineering Center

Materials Research Science and Engineering

Center

$5-15-2005$

\title{
Role of interface bonding in spin-dependent tunneling (invited)
}

Evgeny Y. Tsymbal

University of Nebraska-Lincoln, tsymbal@unl.edu

Kirill D. Belashchenko

University of Nebraska - Lincoln, belashchenko@unl.edu

Follow this and additional works at: https://digitalcommons.unl.edu/mrsecfacpubs

Part of the Materials Science and Engineering Commons

Tsymbal, Evgeny Y. and Belashchenko, Kirill D., "Role of interface bonding in spin-dependent tunneling (invited)" (2005). Faculty Publications: Materials Research Science and Engineering Center. 41.

https://digitalcommons.unl.edu/mrsecfacpubs/41

This Article is brought to you for free and open access by the Materials Research Science and Engineering Center at DigitalCommons@University of Nebraska - Lincoln. It has been accepted for inclusion in Faculty Publications:

Materials Research Science and Engineering Center by an authorized administrator of DigitalCommons@University of Nebraska - Lincoln. 


\title{
Role of interface bonding in spin-dependent tunneling (invited)
}

\author{
E. Y. Tsymbal ${ }^{\text {a) }}$ and K. D. Belashchenko \\ Department of Physics and Astronomy and Center for Materials Research and Analysis, \\ University of Nebraska, Lincoln, Nebraska 68588
}

(Presented on 8 November 2004; published online 5 May 2005)

\begin{abstract}
Measured positive values of the spin polarization of the tunneling current from $3 d$ ferromagnetic metals are commonly explained by the dominant $s$-electron contribution based on symmetry considerations for bulk materials, ignoring the influence of the interfaces. In this work, three different models are considered which suggest that the spin polarization is primarily determined by the electronic and atomic structures of the ferromagnet/insulator interfaces rather than by the bulk properties. A simple tight-binding model demonstrates that the existence of interface states and their contribution to the tunneling current depend on the degree of hybridization between the orbitals on metal and insulator atoms. The decisive role of the interface bonding is further supported by considering spin-dependent tunneling from oxidized Co surfaces through vacuum and in $\mathrm{Co} / \mathrm{Al}_{2} \mathrm{O}_{3} / \mathrm{Co}$ tunnel junctions within the first-principles Green's-function approach. For the oxidized Co surface it is found that the $\mathrm{Co}-\mathrm{O}$ bonding at the surface removes the conducting orbitals forming the bulk Bloch states from the Fermi level, creating an additional tunneling barrier for minority-spin electrons. For the $\mathrm{Co} / \mathrm{Al}_{2} \mathrm{O}_{3} / \mathrm{Co}$ junctions, two types of the interface $\mathrm{O}$ atoms are distinguished: those which saturate $\mathrm{Al}$ bonds and those which are adsorbed by Co. The latter bind strongly to Co creating interface states which enhance the tunneling current in the majority-spin channel. In both cases, the spin polarization changes sign and becomes positive, evidencing the crucial role of the interface structure and bonding. (C) 2005 American Institute of Physics. [DOI: 10.1063/1.1851415]
\end{abstract}

\section{INTRODUCTION}

Tunneling magnetoresistance (TMR) is a phenomenon, which is observed in magnetic tunnel junctions (MTJs) representing two ferromagnetic films separated by a thin insulating barrier layer (for a recent review on TMR, see Ref. 1). The essence of TMR is the dependence of the tunneling current on the relative orientation of the magnetizations of the two ferromagnetic layers. Large reproducible values of TMR (Ref. 2) garnered much attention due to possible applications in nonvolatile random access memories and next-generation magnetic-field sensors.

TMR is a consequence of spin-dependent tunneling (SDT), which is an imbalance in the electric current carried by up- and down-spin electrons tunneling from a ferromagnet through a tunneling barrier. SDT was discovered by Tedrow et al., ${ }^{3}$ who used superconducting layers to detect the spin polarization (SP) of the tunneling current originating from various ferromagnetic electrodes across an alumina barrier. ${ }^{4}$ These experiments found a positive SP for all ferromagnetic $3 d$ metals. This fact was later explained by Stearns, ${ }^{5}$ who assumed that the most dispersive bands provide essentially all the tunneling current. Based on this argument and using a realistic band structure of $\mathrm{Fe}$ and $\mathrm{Ni}$, Stearns was able to explain experimental values (measured at that time) of the SP for these ferromagnets. Despite the success of Stearns' idea, this model did not provide a clear understanding of the origin of the dominance of the "itinerant" electrons in transport properties.

${ }^{a)}$ Electronic mail: tsymbal@unl.edu
More recent theoretical studies provided a new insight into the phenomenon of SDT. It was stated that the expected spin dependence of the tunneling current can be deduced from the symmetry of the Bloch states in the bulk ferromagnetic electrodes and the complex band structure of the insulator. ${ }^{6,7}$ By identifying those bands in the electrodes that are coupled efficiently to the evanescent states decaying most slowly in the barrier one can make conclusions about the SP of the conductance. It was emphasized that for a broad class of insulating materials the states which belong to the identity representation should have minimum decay rates. This representation is comparable to the $s$ character, suggesting that $s$ bands should be able to couple most efficiently across the interface and decay most slowly in the barrier. For $\mathrm{Fe}, \mathrm{Co}$, and $\mathrm{Ni}$ ferromagnets the majority-spin states at the Fermi energy have more $s$ character than the minority-spin states, which tend to have mainly $d$ character. Thus, the majority conductance is expected to be greater than the minority conductance, resulting in a slower decay with barrier thickness for the former. These conclusions are expected to be valid for MTJs with an $\mathrm{Al}_{2} \mathrm{O}_{3}$ barrier which is consistent with the experimental observations. ${ }^{4}$ They are also consistent with the earlier hypothesis by Stearns. ${ }^{5}$

Unfortunately, this plausible explanation of the origin of the positive SP observed experimentally has a number of deficiencies. First, it assumes that the barrier is sufficiently thick so that only a small focused region of the surface Brillouin zone contributes to the tunneling current. For realistic MTJs with a barrier thickness of about $1 \mathrm{~nm}$ this assumption is usually unjustified. Moreover, for amorphous barriers such as alumina where the transverse wave vector is not con- 
served in the process of tunneling, the entire surface Brillouin zone might contribute almost equally to the conductance. Second, despite the presence of certain selection rules for tunneling, there is no general rule preventing the Bloch states composed mostly of the $d$ orbitals from tunneling through the barrier states with no $d$ orbitals. Symmetry strictly forbids tunneling only in special geometries for special values of the wave vector. Finally, symmetry considerations alone applied to bulk materials are not always sufficient to predict the SP. It is critical to take into account the electronic structure of the ferromagnet/barrier interfaces which, as it was shown experimentally, ${ }^{8-10}$ controls SDT.

An important mechanism by which the interfaces affect the SP of the conductance is the bonding between the ferromagnetic electrodes and the insulator. ${ }^{11}$ The interface bonding determines the effectiveness of transmission across the interface which can be different for electrons of different character (and/or symmetry) carrying an unequal SP. Also the interface bonding might be responsible for the appearance of the interface states which, as was predicted theoretically, ${ }^{12}$ affect the conductance dramatically. Experimentally, the effect of bonding at the ferromagnet/insulator interface was proposed to explain the inversion of the SP of tunneling electrons from $\mathrm{Co}$ across a $\mathrm{SrTiO}_{3}$ barrier. ${ }^{13}$ The bonding mechanism was also put forward to explain positive and negative values of TMR depending on the applied voltage in MTJs with $\mathrm{Ta}_{2} \mathrm{O}_{5}$ and $\mathrm{Ta}_{2} \mathrm{O}_{5} / \mathrm{Al}_{2} \mathrm{O}_{3}$ barriers, ${ }^{14}$ and to elucidate the inversion of TMR observed in Co-contacted multiwalled carbon nanotubes. ${ }^{15}$ Theoretically, a strong sensitivity of the magnitude of TMR to the $s p-d$ mixing at the ferromagnet/alumina interface was predicted in the presence of imperfectly oxidized $\mathrm{Al}$ or $\mathrm{O}$ ions. ${ }^{16}$ It was found that oxygen deposited on the $\mathrm{Fe}(001)$ surface reverses the SP of the density of states (DOS) in vacuum due to the strong exchange splitting of the antibonding oxygen states. ${ }^{17}$ It was predicted that an atomic layer of iron oxide at the interface between $\mathrm{Fe}$ and $\mathrm{MgO}$ layers greatly reduces TMR in $\mathrm{Fe} / \mathrm{MgO} / \mathrm{Fe}$ junctions due to the bonding between $\mathrm{Fe}$ and O. ${ }^{18}$

In view of all these experimental and theoretical findings, it would be very surprising to expect that there is a general rule which determines the tunneling SP, entirely based on the bulk properties of the ferromagnet and the insulator. It is more likely that the actual atomic structure of the interface and the strength and the type of the chemical bonding between the atoms of the metal and the insulator determine the SP. Moreover, the sensitivity of the SP to the interface bonding suggests that it is very unlikely that the above arguments based on the dominant $s$-electron tunneling would be justified for tunneling in real MTJ with the alumina barrier.

In this paper, we consider three different models which suggest that the SP is primarily determined by the electronic and atomic structures of the ferromagnet/insulator interfaces rather than by the bulk properties. In Sec. II, we consider a simple tight-binding model which demonstrates that the interface potentials and hybridization essentially control the conductance and its transverse momentum dependence. In Sec. III, we discuss SDT from oxidized Co surfaces through

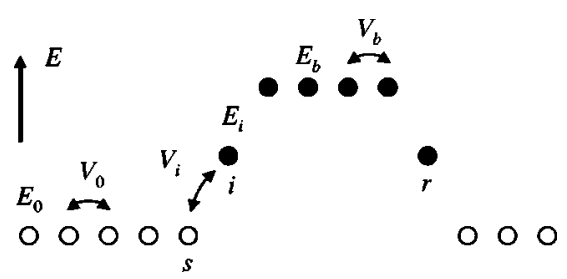

FIG. 1. Geometry of a tunnel junction within a one-dimensional tightbinding model. The open circles denote atoms in the leads and the solid circles denote atoms in the insulating barrier. Vertical positions of the atoms reflect the on-site atomic energies. Parameters of the model are described in text.

vacuum. ${ }^{19}$ We demonstrate that one monolayer of oxygen placed on the fcc $\mathrm{Co}(111)$ surface creates a spin-filter effect due to the $\mathrm{Co}-\mathrm{O}$ bonding. This reverses the sign of the SP from negative for the clean Co surface to positive for the oxidized Co surface. In Sec. IV, we consider SDT in $\mathrm{Co} / \mathrm{Al}_{2} \mathrm{O}_{3} / \mathrm{Co}$ junctions. ${ }^{20}$ We show that there might be two types of the interface $\mathrm{O}$ atoms: those which saturate $\mathrm{Al}$ bonds and those which are adsorbed by Co. The latter bind strongly to Co creating interface states which enhance the tunneling current in the majority-spin channel, thereby reversing the SP.

\section{A SIMPLE TIGHT-BINDING MODEL}

In order to illustrate the decisive effect of the interface bonding on tunneling properties, we consider, first, a onedimensional (1D) single-band tight-binding model. Figure 1 shows geometry of the system which represents a 1D tunnel junction with two metal leads separated by an insulating barrier layer. The left lead consists of a semi-infinite atomic chain with all sites having the same atomic energy levels $E_{0}$ and nearest-neighbor hopping integrals $V_{0}$. The chain is terminated at a site $s$ coupled to the interfacial site $i$ with a hopping integral $V_{i}$. The site $i$ has an atomic level $E_{i}$ and may correspond either to the surface atom of the electrode, or to the nearest barrier site, or to an interfacial "adsorbate." In each of these situations, the parameters $E_{i}$ and $V_{i}$ are determined by interfacial charge transfer and bonding. The insulator is coupled to the right lead, as shown in Fig. 1, the properties of which are not of interest to us. The aim of this simple model is to understand the influence of the bonding $V_{i}$ and the electronic potential $E_{i}$ at the left interface on tunneling conductance.

To simplify the description we assume that the Fermi level $E_{F}$ lies well below the bottom of the insulator conduction band, which is the case if the hopping integral $V_{b}$ between the nearest-neighbor sites in the barrier layer is much less than the barrier height, $V_{b} \ll\left|E_{b}-E_{F}\right|$. In this limit of a high potential barrier the conductance per spin is given by ${ }^{21}$

$$
G(E)=\frac{4 \pi^{2} e^{2}}{h} V_{b}^{2} N_{i}(E) \exp (-2 \kappa a N) N_{r}(E),
$$

where $G(E)$ is the conductance at a given energy $E$ (Fermi energy), $\kappa=1 / a \ln \left[\left(E_{b}-E / V_{b}\right)\right]$ is the decay constant, $a$ is the lattice parameter, $N+2$ is the total number of atoms in the insulator including two atoms at the interfaces, and $N_{i}(E)$ and $N_{r}(E)$ are the local DOS at the interface sites $i$ and $r$, 
respectively, unperturbed by the coupling $V_{b}$ to the adjacent sites in the insulator. We assumed in Eq. (1) that sites $i$ and $r$ are coupled to the insulator by hopping integrals $V_{b}$, but this assumption is immaterial as long as these couplings are small compared to the barrier height.

In Eq. (1) the only quantity which depends on the parameters characterizing the left interface, $V_{i}$ and $E_{i}$, is the local DOS $N_{i}(E)$. Therefore, below we focus on the calculation of this quantity. We use dimensionless variables $\varepsilon=(E$ $\left.-E_{0}\right) / V_{0}, \varepsilon_{i}=\left(E_{i}-E_{0}\right) / V_{0}, w=V_{i} / V_{0}$, and $\rho(\varepsilon)=V_{0} N(E)$ for all densities of states. The bulk band dispersion is given by

$$
\boldsymbol{\epsilon}(k)=-2 \cos k a,
$$

where $k$ is the wave vector. From this expression we find the bulk DOS

$$
\rho_{0}(\varepsilon)=\frac{1}{\pi \sqrt{4-\varepsilon^{2}}} .
$$

In order to obtain the local DOS at the interface atom, $\rho_{i}(\varepsilon)$, we use Dyson's equation. First, we find the retarded surface Green's function of the homogeneous semi-infinite wire at site $s$, and then attach the interface atom $i$. For a homogeneous chain of atoms and for $\varepsilon$ lying within the bulk band, $|\varepsilon|<2$, we obtain

$$
g_{s}(\varepsilon)=\frac{1}{2}\left(\varepsilon-i \sqrt{4-\varepsilon^{2}}\right)
$$

and

$$
\rho_{s}(\varepsilon)=\frac{\sqrt{4-\varepsilon^{2}}}{2 \pi} .
$$

If an additional atom of on-site energy $\varepsilon_{i}$ is attached at the end of this semi-infinite chain and coupled to its neighboring atom $s$ with bond integral $w$, for $|\varepsilon|<2$ the on-site Green's function is given by

$$
g_{i}(\varepsilon)=\left[\varepsilon-\varepsilon_{i}-w^{2} g_{s}(\varepsilon)\right]^{-1} .
$$

The Green's function for $|\varepsilon|>2$ may be obtained by analytic continuation via the upper half-plane. The interface DOS in Eq. (1) is then simply

$$
\rho_{i}(\varepsilon)=-\operatorname{Im} g_{i}(\varepsilon+i 0) / \pi .
$$

The bulk and surface DOS of the homogeneous semiinfinite system given by Eqs. (3) and (5) behave very differently. At the band edges the bulk DOS diverges, while the surface DOS vanishes. This behavior of the surface DOS may be understood as a crossover to the free-electron regime. Indeed, in the long-wavelength limit, $k a \ll 1$, corresponding to the band bottom the discrete structure of the atomic chain becomes irrelevant, and the free-electronlike wave function develops a node at the surface. This also applies to the band top where the free-electronlike wave function may be obtained by a gradient expansion around the $k a=\pi$ state. The criterion of the free-electronlike behavior is a vanishing group velocity.

However, the interface bonding can easily destroy all the similarities to the free-electron system in the behavior of the local DOS. Indeed, the structure of the wave function near the interface cannot be fully described by the free-electron

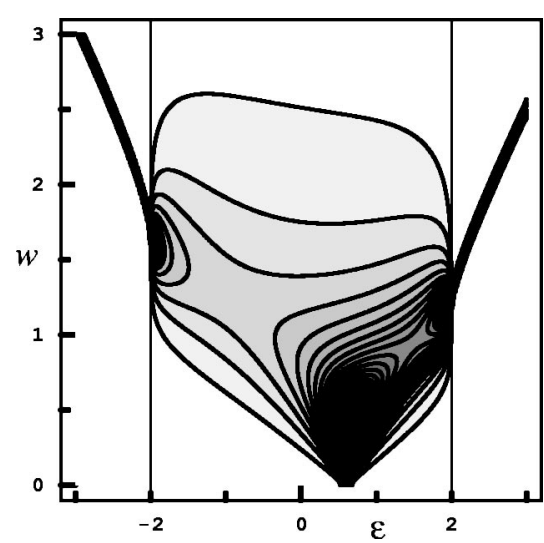

FIG. 2. Contour plot of the interface DOS, $\rho_{i}$, as a function of energy $\varepsilon$ and interface bonding strength $w$ for the tight-binding model described in the text. The interface atom level is $\varepsilon_{i}=0.6$. An imaginary part of 0.001 was added to $\varepsilon$ to resolve the localized surface states emerging from the continuum at large $w$. The contour lines correspond to values starting at 0.05 and increasing with the step of 0.05 . The vertical lines at $\varepsilon= \pm 2$ show the bulk band edges. The gray level of shading increases with the surface DOS value.

gradient expansion. To illustrate this statement, we have plotted in Fig. 2 the interface DOS as a function of energy $\varepsilon$ and interface bonding parameter $w$ for a rather arbitrary choice of $\varepsilon_{i}=0.6$. It is seen that the interface DOS may be strongly enhanced. For strong coupling $w$ this enhancement occurs at band edges. In this case localized states emerge from the continuum. These states correspond to bonding and antibonding orbitals formed by the atom $s$ and its nearest neighbor $i$, modified by the interaction with the bulk band. For weak coupling $w$ the interface DOS is enhanced around the interface atom level $\varepsilon_{i}=0.6$. As $w$ decreases starting from large values and as the localized level approaches the band edge and then enters it, becoming a surface resonance, the interface DOS near this edge is strongly enhanced. Thus, we see that the magnitude of the interface DOS and, consequently, the magnitude of the conductance are essentially controlled by the strength of bonding and atomic potential at the interface.

This observation clearly shows that it is impossible to predict the features of the tunneling conductance based on free-electronlike models with no interfacial specificity. The strong dependence of the interface DOS on the interface parameters suggests that the SP of the tunneling current must also be very sensitive to the strength of bonding and atomic potential at the interface due to a different electronic structure for majority- and minority-spin electrons.

To illustrate these points further, we add the in-plane dispersion to our tight-binding model by considering a simple cubic lattice with nearest-neighbor hopping. For simplicity we assume that all hopping integrals for bonds parallel to the surface are equal to $V_{0}$. The integrals for perpendicular bonds between the interface $i$ and surface $s$ layers are assumed to be equal to $V_{i}$, and we again denote $w=V_{i} / V_{0}$.

In this three-dimensional (3D) model, the in-plane component of the wave vector $\mathbf{k}_{\|}$is conserved and we can use it as a quantum number. The Hamiltonian in this representation is identical to the 1D one considered above, except that it now acquires an additive in-plane dispersion term 


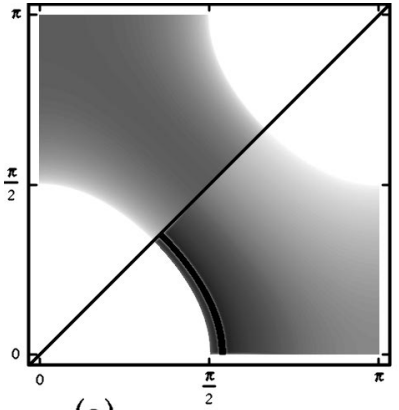

(a)

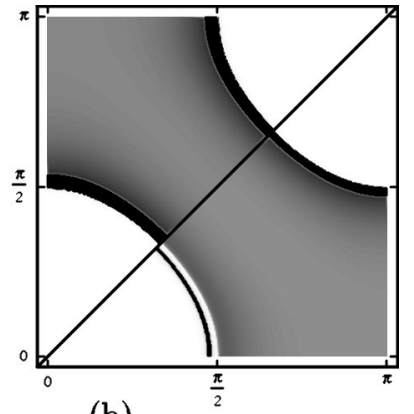

(b)
FIG. 3. Plots of the interface DOS $\rho_{i}$ in a quarter of the surface Brillouin zone for the 3D model. The parameter $w$ is equal to 1 in panel (a) and 1.5 in panel (b). The Fermi level is $\varepsilon_{F}=0$. The top left corner of each panel shows $\rho_{i}$ for $\varepsilon_{i}=0$; the bottom right corner, for $\varepsilon_{i}=0.6$. The gray contrast increases with the interface DOS value. The white color corresponds to zero $\rho_{i}$; the black color to 0.5 (a) and to 0.3 (b).

$$
\varepsilon_{\|}=-2\left(\cos k_{x} a+\cos k_{y} a\right),
$$

which does not depend on the layer number (here we choose the $z$ axis normal to the surface). Therefore, all formulas obtained above hold true as long as we replace $\varepsilon$ by $\varepsilon_{\perp}=\varepsilon$ $-\varepsilon_{\|}$(the same holds for Fig. 2).

Figure 3 shows plots of the surface DOS as a function of $\mathbf{k}_{\|}$with the Fermi level chosen at $\varepsilon_{F}=0$, for different combinations of $w$ and $\varepsilon_{i}$. Figure 3(a) shows $\rho_{i}$ for $w=1$, and Fig. 3(b), for $w=1.5$. In each figure, the top left corner shows $\rho_{i}$ for $\varepsilon_{i}=0$, and the bottom right corner for $\varepsilon_{i}=0.6$. It is clearly seen that the shape of the surface DOS is very different for the four cases. For $w=1, \varepsilon_{i}=0$ (no surface perturbations) the system behaves similar to the free-electron limit. However, in all other cases the spectral weight is strongly displaced toward one of the edges of the Fermi-surface projection (or both edges). For example, at $w=1, \varepsilon_{i}=0.6$ smaller values of $k_{x}$ and $k_{y}$ correspond to lower $\varepsilon_{\|}$and hence higher $\varepsilon_{\perp}$ where, as seen in Fig. 2, the surface DOS has a maximum.

The above example shows that seemingly small variations in the atomic potentials and hopping integrals near the interface may have a very strong and unexpected effect on the shape of the interface DOS and, hence, on the conductance. ${ }^{22}$ Since such variations are common in real materials, the behavior of the interface DOS for bands formed by localized $3 d$ states in transition metals should be very sensitive to the interfacial structure and bonding. In particular, one should expect that at least some of the edges of the Fermi-surface projection will exhibit a strong surface DOS enhancement, contrary to the predictions of the free-electron model. Also, the interface bonding might lead to the formation of the interface states, affecting dramatically the SP of the tunneling current. As we will see in Secs. III and IV, these effects, indeed, occur in real MTJs which are more complicated compared to that described by the simple tightbinding model due to the presence of many hybridized bands with complex dispersion.

\section{TUNNELING FROM OXIDIZED CO SURFACE}

In Ref. 19 we calculated the transmission functions for clean and oxidized $\mathrm{Co}(111) /$ vacuum/Al MTJs using the
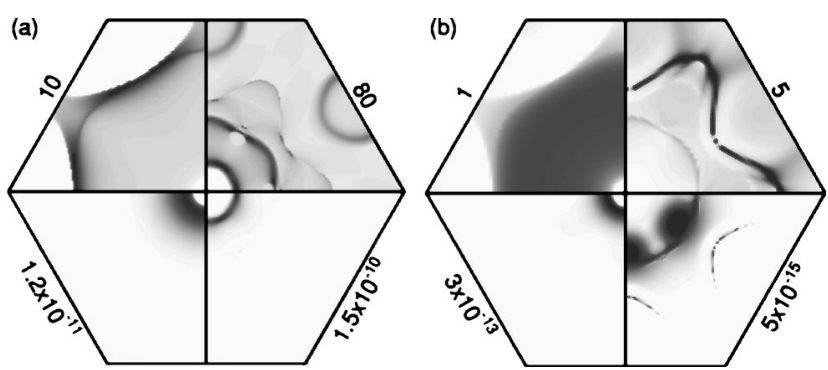

FIG. 4. Results of first-principles calculations for $\mathrm{Co}(111) / \mathrm{vacuum} / \mathrm{Al}$ MTJs. (a) Clean Co surface and (b) Co surface with an adsorbed O monolayer. Each figure shows the interface Brillouin zone split in four sections. The top left (right) section displays the majority-spin (minority-spin) $\mathbf{k}_{\|}$-resolved DOS at the surface layer of Co in (a) and for the O layer in (b). The bottom left (right) section shows the majority-spin (minority-spin) $\mathbf{k}_{\|}$-resolved conductance for the given MTJ. The gray level varies from white (zero value) to black (value shown next to each frame section). The units are $\mathrm{Ry}^{-1} /$ atom for DOS and $e^{2} / h$ for the conductances.

principal-layer Green's-function approach ${ }^{23,24}$ and the newly introduced surface transmission functions (STFs) characterizing the penetration of the Bloch states into the barrier. This approach involves metal-induced DOS inside the barrier and thereby properly takes into account all selection rules appearing in the actual multiband system. The STF approach and its validity region are similar in spirit to Eq. (1).

Figure 4 shows the results of calculations for MTJs with the clean (a) and oxidized (b) Co surface. The oxidation consists of depositing a monolayer of $\mathrm{O}$ atoms in the structurally relaxed threefold hollow-site positions above the subsurface Co layer. We observe the drastic effect of surface bonding on the surface DOS, the tunneling transmission and its spin polarization. For the clean surface, the tunneling conductance has a large negative SP of $-60 \%$. Note that two Fermi-surface edges in the minority channel are strongly emphasized in the DOS [the top right section in Fig. 4(a)]. One of them corresponding to smaller $\kappa\left(\mathbf{k}_{\|}\right)$dominates in the conductance [the bottom right section in Fig. 4(a)]. This resembles the situation described in Sec. II and demonstrates the tight-bindinglike character of the minority-spin $3 d$ band, as opposed to the free-electronlike majority-spin band. The oxidation results in the appearance of the strong covalent bonds between $\mathrm{Co}$ and $\mathrm{O}$ at the surface, and the antibonding states lying around the Fermi level. The intersection of this antibonding surface (resonant) band with the Fermi level is pronounced in the top right section of Fig. 4(b). It is only seen for minority-spin electrons because of a selection rule that prevents them from mixing with bulk states in this channel (for details, see Ref. 19). The minority-spin surface states lie rather far from the center of the surface Brillouin zone, and hence are suppressed by the vacuum decay, as seen in the bottom right section of Fig. 4(b). At the same time, the interface bonding removes the spectral weight from the center of the Brillouin zone. As a result, the tunneling conductance for the MTJ with the oxidized Co surface is fully dominated by the majority-spin channel, resulting in SP of almost $+100 \%$.

\section{TUNNELING IN Co/ $\mathrm{Al}_{2} \mathrm{O}_{3} / \mathrm{Co}$ JUNCTIONS}

Spin-dependent tunneling in $\mathrm{Co} / \mathrm{Al}_{2} \mathrm{O}_{3} / \mathrm{Co}$ MTJs exhibits features similar to the $\mathrm{Co} /$ vacuum/Al system. ${ }^{20}$ These fea- 


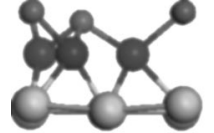

(a)

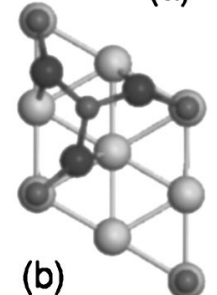

(b)

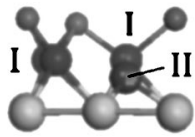

(c)

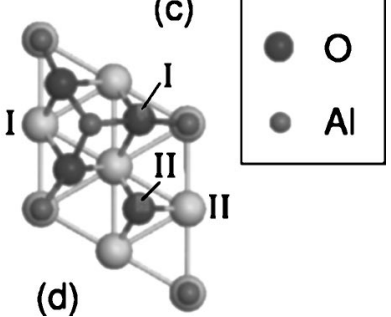

FIG. 5. Interfacial structure for model 1 ( $a$ and $b$ ) and model 2 ( $c$ and d) of the $\mathrm{Co} / \mathrm{Al}_{2} \mathrm{O}_{3}$ MTJ. Panels (a) and (c) show "front" views from a direction normal to the threefold axis; panels (b) and (d) show "top" views along the threefold axis. There are two types of $\mathrm{Co}$ and $\mathrm{O}$ atoms at the interface for model 2: three $\mathrm{O}$ (I) atoms, one $\mathrm{O}(\mathrm{II})$ atom, one $\mathrm{Co}(\mathrm{I})$ atom, and three $\mathrm{Co}(\mathrm{II})$ atoms per unit cell.

tures are revealed by first-principles calculations for the two fully relaxed structural models. The interfacial structure for these models is shown in Fig. 5. Both models have (111)oriented fcc $\mathrm{Co}$ electrodes. Model 1 represents the O-terminated $\mathrm{Co} / \mathrm{Al}_{2} \mathrm{O}_{3} / \mathrm{Co}$ structure studied previously. ${ }^{25}$ Model 2 adds an additional $\mathrm{O}$ atom in the threefold hollow site (this $\mathrm{O}$ atom and the neighboring Co atoms are labeled "II" in Figs. 5(c) and 5(d); the other O and surface Co atoms are labeled "I"). O(II) atoms form strong bonds with $\mathrm{Co}$ (II) atoms similar to the oxidized Co surface. The structures of both models were relaxed using the pseudopotential planewave method ${ }^{26}$ within the generalized gradient approximation.

It is seen in Fig. 5(c) that the structural sites occupied by $\mathrm{O}(\mathrm{I})$ and $\mathrm{O}(\mathrm{II})$ atoms are very dissimilar. $\mathrm{O}(\mathrm{II})$ atoms lie much closer to the Co surface compared to O(I) atoms; in fact, within a few hundredths of an angstrom these sites are identical to the $\mathrm{O}$ adsorption sites for monolayer coverage. ${ }^{19}$ Thus, O(II) atoms are much stronger coupled with Co than with $\mathrm{O}$ (I) and $\mathrm{Al}$ atoms. Qualitatively, $\mathrm{O}(\mathrm{II})$ atoms may be regarded as "Co adsorbates," while $\mathrm{O}(\mathrm{I})$ atoms, as " $\mathrm{Al}_{2} \mathrm{O}_{3}$ terminating." This distinction is evident in the local DOS for the interfacial atoms shown in Fig. 6. The bonding states
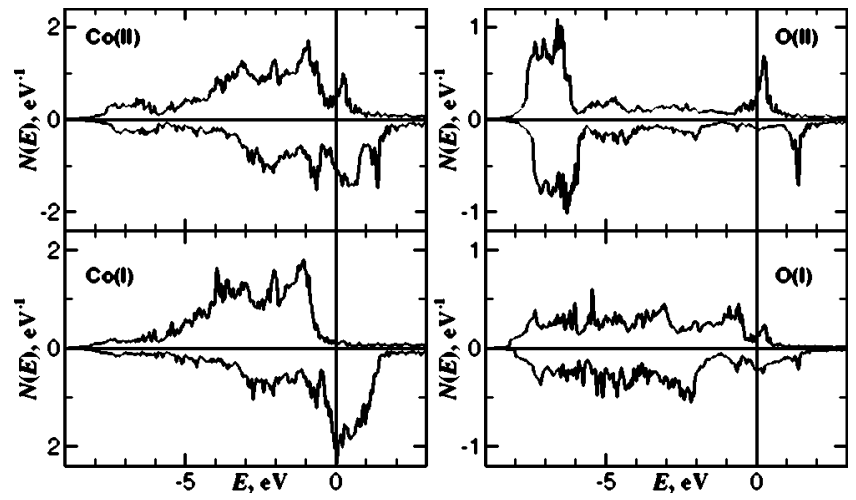

FIG. 6. Local densities of states for interfacial atoms in model 2 for majority (top panels) and minority (bottom panels) spins. In each figure, top half shows the majority-spin DOS, and bottom half, the minority-spin DOS per atom. The vertical line denotes the position of the Fermi level.
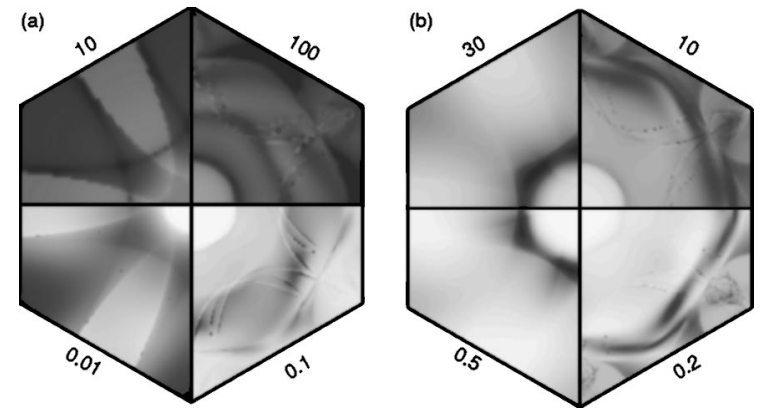

FIG. 7. Same as in Fig. 4, but for $\mathrm{Co} / \mathrm{Al}_{2} \mathrm{O}_{3} / \mathrm{Co}$ MTJs. (a) model 1 and (b) model 2. The DOS in top sections is shown for the interface layer of Co in (a) and for the $\mathrm{O}(\mathrm{II})$ atom in (b).

formed by $\mathrm{Co}(\mathrm{II})$ and $\mathrm{O}(\mathrm{II})$ atoms are located just below the bulk Co $3 d$ band; the exchange-split antibonding states are just above the Fermi level. The local DOS for Co(I) atoms is quite similar to bulk Co, while the local DOS for $\mathrm{O}(\mathrm{I})$ atoms shows a small but notable "echo" of the $\mathrm{Co}(\mathrm{II})-\mathrm{O}$ (II) antibonding states.

O(II) atoms in model 2 provide additional current pathways compared to model 1. As follows from Fig. 6, these pathways should have a strong positive SP because of the large spin asymmetry in the local O(II) DOS at the Fermi level. This is confirmed by the calculations of the local $\mathbf{k}_{\|}$-resolved DOS and tunneling conductance which are shown in Figs. 7(a) and 7(b). The presence of the type-II O atoms reverses sign of the SP making it $+32 \%$ in model 2 compared to $-70 \%$ in model 1 . As seen in the figure, the main effect consists in the significant (20-fold) enhancement of the majority-spin conductance (compared to the twofold increase in the minority-spin conductance). This is the consequence of the majority-spin antibonding $\mathrm{Co}(\mathrm{II})-\mathrm{O}$ (II) states, which dominate in the $\mathbf{k}_{\|}$-resolved DOS and conductance [left sections in Fig. 7(b)]. The corresponding minority-spin states lie more than $1 \mathrm{eV}$ above the Fermi level due to exchange splitting, and hence do not contribute to the conductance.

\section{CONCLUSIONS}

This paper emphasizes the crucial effect of interface bonding on spin-dependent tunneling in magnetic tunnel junctions. Consideration of a simple single-band model shows that the free-electronlike description of the tunneling conductance breaks down if the conducting bands have a tight-binding character. A modest modification of the tightbinding parameters at the interface can strongly affect the surface DOS, and hence the conductance.

First-principle calculations provide realistic examples for $\mathrm{Co} /$ vacuum/Al and $\mathrm{Co} / \mathrm{Al}_{2} \mathrm{O}_{3} / \mathrm{Co}$ tunnel junctions, where multiband effects are important. For the vacuum barrier it is shown that depositing a monolayer of oxygen on the $\mathrm{Co}(111)$ surface reverses the spin polarization from $-60 \%$ to almost $+100 \%$ due to the formation of surface bands that mix well with majority-spin Bloch states but create an additional tunneling barrier for minority-spin Bloch states. For the $\mathrm{Al}_{2} \mathrm{O}_{3}$ barrier, we demonstrate that a somewhat similar effect is produced by interfacial adsorption of oxygen at the 
$\mathrm{Co} / \mathrm{Al}_{2} \mathrm{O}_{3}$ interface. Contrary to the $\mathrm{Co} /$ vacuum/Al MTJ, however, the spin dependence in this case is related to the exchange splitting of the antibonding $\mathrm{Co}-\mathrm{O}$ states. These results suggest a possible explanation of the experimentally observed positive spin polarization in alumina-based MTJs.

\section{ACKNOWLEDGMENTS}

We are thankful to M. van Schilfgaarde, I. I. Oleynik, D. A. Stewart, and S. S. Jaswal, who made significant contributions to the results presented here. This work was supported by NSF Grants Nos. (DMR-0203359 and MRSEC DMR0213808) and the Nebraska Research Initiative.

${ }^{1}$ E. Y. Tsymbal, O. N. Mryasov, and P. R. LeClair, J. Phys.: Condens. Matter 15, R109 (2003).

${ }^{2}$ J. S. Moodera, L. R. Kinder, T. M. Wong, and R. Meservey, Phys. Rev. Lett. 74, 3273 (1995).

${ }^{3}$ P. M. Tedrow, R. Meservey, and P. Fulde, Phys. Rev. Lett. 25, 1270

(1970); P. M. Tedrow and R. Meservey, ibid. 26, 192 (1971); Phys. Rev.

B 7, 318 (1973).

${ }^{4}$ R. Meservey and P. M. Tedrow, Phys. Rep. 238, 173 (1994).

${ }^{5}$ M. B. Stearns, J. Magn. Magn. Mater. 5, 1062 (1977).

${ }^{6}$ J. M. MacLaren, X.-G. Zhang, W. H. Butler, and X. D. Wang, Phys. Rev. B 59, 5470 (1999).

${ }^{7}$ Ph. Mavropoulos, N. Papanikolaou and P. H. Dederichs, Phys. Rev. Lett. 85, 1088 (2000)

${ }^{8}$ P. LeClair, H. J. M. Swagten, J. T. Kohlhepp, R. J. M. van de Veerdonk, and W. J. M. de Jonge, Phys. Rev. Lett. 84, 2933 (2000)

${ }^{9}$ P. LeClair, J. T. Kohlhepp, H. J. M. Swagten, and W. J. M. de Jonge, Phys. Rev. Lett. 86, 1066 (2001).

${ }^{10}$ P. LeClair, B. Hoex, H. Wieldraaijer, J. T. Kohlhepp, H. J. M. Swagten, and W. J. M. de Jonge, Phys. Rev. B 64, 100406 (2001).

${ }^{11}$ E. Y. Tsymbal and D. G. Pettifor, J. Phys.: Condens. Matter 9, L411 (1997).

${ }^{12}$ O. Wunnicke, N. Papanikolaou, R. Zeller, P. H. Dederichs, V. Drchal, and J. Kudrnovský, Phys. Rev. B 65, 064425 (2002).

${ }^{13}$ J. M. De Teresa, A. Barthélémy, A. Fert, J. P. Contour, R. Lyonnet, F. Montaigne, P. Seneor, and A. Vaures, Phys. Rev. Lett. 82, 4288 (1999); A. Barthélémy, A. Fert, J. P. Contour, F. Montaigne, and P. Seneor, Science 286, 507 (1999)

${ }^{14}$ M. Sharma, S. X. Wang, and J. H. Nickel, Phys. Rev. Lett. 82, 616 (1999).

${ }^{15}$ B. Zhao, I. Mönch, T. Mühl, H. Vinzelberg, and C. M. Schneider, J. Appl. Phys. 91, 7026 (2002).

${ }^{16} \mathrm{H}$. Itoh and J. Inoue, Surf. Sci. 493, 748 (2001).

${ }^{17}$ E. Y. Tsymbal, I. I. Oleinik, and D. G. Pettifor, J. Appl. Phys. 87, 5230 (2000).

${ }^{18}$ X.-G. Zhang, W. H. Butler, and A. Bandyopadhyay, Phys. Rev. B 68 , 092402 (2003)

${ }^{19}$ K. D. Belashchenko, E. Y. Tsymbal, M. van Schilfgaarde, D. A. Stewart, I. I. Oleynik, and S. S. Jaswal, Phys. Rev. B 69, 174408 (2004).

${ }^{20}$ K. D. Belashchenko, E. Y. Tsymbal, I. I. Oleynik, and M. van Schilfgaarde submitted to Phys. Rev. B.

${ }^{21}$ J. Mathon, Phys. Rev. B 56, 11810 (1999)

${ }^{22}$ The conductance calculation in the 3D case requires the summation over transverse wave vectors $\mathbf{k}_{\|}$and therefore the knowledge of the $\mathbf{k}_{\|}$dependence of the decay constant $\kappa\left(\mathbf{k}_{\|}\right)$and the local DOS $N_{r}\left(\mathbf{k}_{\|}\right)$.

${ }^{23}$ I. Turek, V. Drchal, J. Kudrnovský, M. Šob, and P. Weinberger, Electronic Structure of Disordered Alloys, Surfaces, and Interfaces (Kluwer, Boston, 1997)

${ }^{24}$ J. Kudrnovský, V. Drchal, C. Blaas, P. Weinberger, I. Turek, and P. Bruno, Phys. Rev. B 62, 15084 (2000).

${ }^{25}$ I. I. Oleinik, E. Y. Tsymbal, and D. G. Pettifor, Phys. Rev. B 62, 3952 (2000).

${ }^{26}$ M. C. Payne, M. P. Teter, D. C. Allan, T. A. Arias, and J. D. Joannopoulos, Rev. Mod. Phys. 64, 1045 (1992) 